\section{Mechanical Ventilation for the Treatment of Severe Excessive Dynamic Airway Collapse}

\section{To the Editor:}

We read with interest the case report by Ismael et $\mathrm{al}^{1}$ describing a patient with Sjögren's syndrome and cystic lung disease who could not be weaned from a ventilator due to severe central excessive dynamic airway collapse (EDAC) of the lower part of the trachea and proximal bronchi. EDAC corresponds to the expiratory bulging of the tracheobronchial wall without known airway structural abnormalities, leading to a decrease of at least $50 \%$ in internal diameter. ${ }^{2}$ It is a rare and underdiagnosed entity, commonly confused with other respiratory diseases such as asthma and COPD. Although noninvasive procedures such as cervicothoracic computed tomography scan on inspiration and expiration may suggest the disorder, the accepted standard method for diagnosis is bronchoscopy. ${ }^{3-7}$

There is no consensus on the best treatment. The authors stated that stenting could resolve the collapsibility in that case. ${ }^{1}$ In our practice, endotracheal or endobronchial stenting is effective when the collapse affects only a small area of the trachea or main bronchi. In some patients with diffuse airway involvement, stenting is frequently useless. Murgu and Colt ${ }^{2}$ proposed an algorithm for the management of EDAC. Preceding invasive measures, the authors recommend the appropriate pharmacologic treatment of comorbidities, including COPD, asthma, and gastroesophageal reflux disease, because they may be predisposing factors for airway collapse. When treatment is not sufficient or the exacerbations become more frequent, noninvasive ventilation (NIV) may be tried. There are reports of significant improvement with CPAP $>6 \mathrm{~cm} \mathrm{H}_{2} \mathrm{O} .{ }^{8}$

We want to report our case of a female patient with scleroderma and a longstanding history of poorly controlled asthma who presented with severe and diffuse EDAC. Bronchoscopy (Fig. 1) showed a striking expiratory bulging $(75-100 \%)$ of the larynx, posterior wall of the trachea, and bronchial tree bilaterally (main, lobar, and segmental bronchi), with some parts of the airway walls making contact. Pulmonary involvement with connectivitis was excluded, and histological examination of bronchial and hypopharynx biopsies was inconclusive

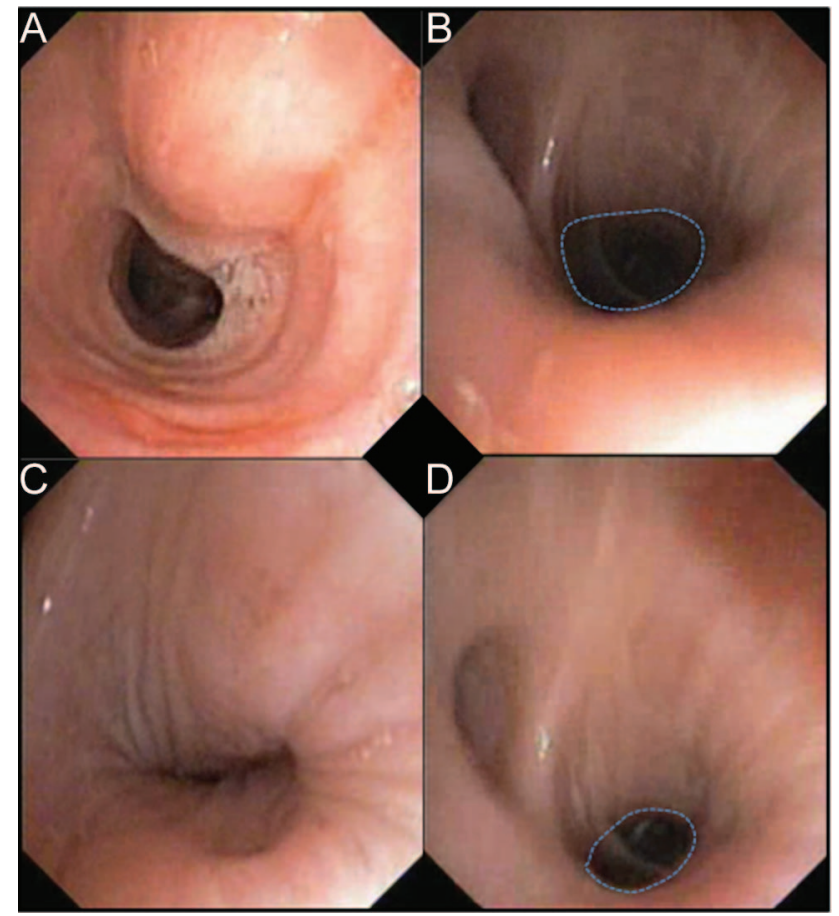

Fig. 1. Bronchoscopic images of the trachea. A: The beginning of the bulging of the posterior wall over its lumen in expiration. B: Intermediate bronchus in inspiration. C: Intermediate bronchus in expiration with complete luminal collapse. D: The diameter of the intermediate bronchus is shown in expiration with the application of CPAP.

(chronic nonspecific inflammation). Following consecutive hospitalizations requiring invasive mechanical ventilation support, the patient started nocturnal CPAP with $10 \mathrm{~cm} \mathrm{H}_{2} \mathrm{O}$ (see Fig. 1).

The patient remained relatively stable for $\sim 1 \mathrm{y}$, when she was again invasively ventilated in 2 successive admissions. Due to weaning difficulties, NIV with bi-level positive airway pressure was introduced after extubation. The patient became increasingly dependent on ventilatory support and gradually began to require continuous NIV with a full-face mask (aiming to prevent skin lesions). At this stage, the patient could not tolerate more than brief periods without NIV, just enough to feed herself. Due to the high degree of dependence on the ventilator and the continued clinical worsening, a tracheotomy was proposed. Her ventilator settings were adjusted according to the presence of a fenestrated inner cannula and cuff inflation. Thus, during the day using the fenestration and a deflated cuff, the parameters were: BiPAP S/T with AVAPS (tidal volume of $500 \mathrm{~mL}$ guaranteed), maximum and minimum inspiratory positive airway pressures of 18 and $13 \mathrm{~cm} \mathrm{H}_{2} \mathrm{O}$, expiratory positive airway pressure of $8 \mathrm{~cm} \mathrm{H} \mathrm{H}_{2} \mathrm{O}$, breath- ing frequency of 12 breaths/min, and inspiratory time of $1.1 \mathrm{~s}$. At night, without the fenestra and with an inflated cuff, the parameters were: BiPAP $\mathrm{S} / \mathrm{T}$, inspiratory positive airway pressure of $14 \mathrm{~cm} \mathrm{H}_{2} \mathrm{O}$, expiratory positive airway pressure of $8 \mathrm{~cm} \mathrm{H}_{2} \mathrm{O}$, breathing frequency of 12 breaths/min, and inspiratory time of $1.1 \mathrm{~s}$. Because the patient was tracheostomized, she had several microbial isolates in bronchial secretions. After a few months of stability, she was again admitted with a new episode of unstoppable cough and respiratory failure. New bronchoscopic examination revealed that the patient had increased tracheal collapsibility with cuff deflation. Keeping the cuff continuously inflated and sedating the patient were the only solutions for ventilation, ending the cough, and improving gas exchange. She finally died due to septic shock with nosocomial pneumonia $\sim 5$ y after diagnosis.

We want to emphasize the need for more research to elucidate the underlying pathogenesis and optimum management of dynamic airway collapse. As highlighted by the 2 cases, this is a condition with significant morbidity and mortality, and treatment 
must be adapted to the severity and extension of the disease.

Helder Novais Bastos MD

Department of Pneumology

Centro Hospitalar de São João

Porto, Portugal

and

Life and Health Sciences Research Institute (ICVS)

School of Health Sciences

University of Minho

Braga, Portugal

and

ICVS/3B's-PT Government Associate

Laboratory

Braga/Guimarães, Portugal

Nélson Teixeira MD

Margarida Redondo MD

Department of Pneumology

Centro Hospitalar de São João

Porto, Portugal

Miguel Gonçalves PhD

Department of Pneumology

Centro Hospitalar de São João

Porto, Portugal

and

Faculty of Medicine

University of Porto

Porto, Portugal

Maria Sucena MD

Department of Pneumology

Centro Hospitalar de São João

Porto, Portugal

The authors have disclosed no conflicts of interest.

DOI: $10.4187 /$ respcare. 03972

\section{REFERENCES}

1. Ismael S, Wermert D, Dang-Tran KD, Venot M, Fagon JY, Diehl JL. Severe excessive dynamic airway collapse in a patient with primary Sjögren's syndrome. Respir Care 2014;59(10):e156-e159.

2. Murgu SD, Colt HG. Tracheobronchomalacia and excessive dynamic airway collapse. Respirology 2006;11(4):388-406.

3. Joosten S, MacDonald M, Lau KK, Bardin P, Hamilton G. Excessive dynamic airway collapse co-morbid with COPD diagnosed using 320-slice dynamic CT scanning technology. Thorax 2012;67(1):95-96.

4. Boiselle PM, Feller-Kopman D, Ashiku S, Weeks D, Ernst A. Tracheobronchomalacia: evolving role of dynamic multislice he- lical CT. Radiol Clin North Am 2003;41(3): 627-636.

5. Baroni RH, Ashiku S, Boiselle PM. Dynamic $\mathrm{CT}$ evaluation of the central airways in patients undergoing tracheoplasty for tracheobronchomalacia. AJR 2005;184(5): 1444-1449.

6. Lee KS, Sun MR, Ernst A, Feller-Kopman D, Majid A, Boiselle PM. Comparison of dynamic expiratory CT with bronchoscopy for diagnosing airway malacia: a pilot evaluation. Chest 2007;131(3):758-764.

7. Loring SH, O'Donnell CR, Feller-Kopman DJ, Ernst A. Central airway mechanics and flow limitation in acquired tracheobronchomalacia. Chest 2007;131(4):1118-1124.

8. Macedo Neto AV, Oliveira HG, Macedo BR, Valle E. Bronchoscopic management of functional airway obstruction. Pulmão RJ 2011;20(2):8-13. Article in Portuguese.

\section{Excessive Dynamic Airway Collapse in the Critical Care Setting}

In Reply:

We read with great interest the letter of Bastos et al ${ }^{1}$ describing both the value and limits of mechanical ventilation for the treatment of severe excessive dynamic airway collapse (EDAC). Their case, in addition to our previously published case $^{2}$ and other reports, raises some important questions with regard to the optimal management of ICU patients with severe EDAC.

First, it is important to diagnose expiratory central airway collapse in an ICU patient with predisposing conditions (such as asthma in the case of Bastos et $\mathrm{al}^{1}$ or Sjögren's syndrome in our case), developing acute respiratory failure with a need for mechanical ventilation, or persistent or difficult weaning from mechanical ventilation. As highlighted by Murgu and Colt, ${ }^{3}$ such a condition is relatively frequent, but one suspects that it is underdiagnosed and poorly managed in some instances. In the critical care setting, bronchoscopy remains the best approach to recognize and assess the degree of narrowing (including its dynamic component, if any). Moreover, it allows us to distinguish between EDAC and tracheobronchomalacia. However, special attention should be given to standardization of the procedure (which could preferentially include the recording of video clips) and the reports. ${ }^{4-6}$ This is of particular importance both for individual patient care and for development of multi-institutional or collaborative studies or registries.
Other diagnostic approaches such as imaging studies or pulmonary function studies can complete the bronchoscopic evaluation, but with some limitations in the critical care setting. Dynamic computed tomography studies are difficult to perform in mechanically ventilated patients, but comparison of end-expiratory and end-inspiratory acquisitions could, in some cases, add additional information, such as in our patient, by the demonstration of a possible expiratory posterior compression of the intermediate bronchus by parenchymal lung cysts. ${ }^{2}$ Impulse oscillometry has recently been found to be a good correlate with clinical symptoms in subjects with central airway obstruction and is able to discriminate between variable and fixed central airway obstruction. ${ }^{7}$ Theoretically, such a method could also be proposed and evaluated in patients on controlled mechanical ventilation.

At the end of the diagnostic process, every effort should be made to objectively describe the morphologies and etiologies of EDAC, as well as the degree of functional impairment, extent of disease, and severity of airway collapse. We suggest using the FEMOS (functional status, extent of abnormality, morphology, origin, severity of airway collapse) classification system developed by Murgu and Colt,${ }^{3}$ both for individual decisions and therapeutic evaluations and for allowing multi-institutional registry participation or collaborative studies.

Finally, for a given patient, all therapeutic option components, including bronchodilators, disease-specific drug therapy if any, positive-pressure ventilation, and airway stenting, should be considered. These should be viewed as complementary to each other. The management algorithms proposed by Murgu and Colt $^{3}$ seem to be an attractive approach.

Jean-Luc Diehl MD Nadia Aissaoui MD PhD Medical Intensive Care Unit Georges Pompidou European Hospital Assistance Publique-Hôpitaux de Paris Paris, France and

Institut National de la Santé et de la Recherche Médicale (INSERM) U 1140

Faculté de Médecine et Faculté de Pharmacie Université Paris Descartes Sorbonne Paris Cité Paris, France 